\title{
BASIC SCIENCE ARTICLE OPEN Increased miR-214 expression suppresses cell migration and proliferation in Hirschsprung disease by interacting with PLAGL2
}

\author{
Liang Wu ${ }^{1}$, Wenzheng Yuan ${ }^{1,3}$, Jinhuang Chen ${ }^{1}$, Zili Zhou ${ }^{1}$, Yan Shu ${ }^{2}$, Jintong $\mathrm{Ji}^{1}$, Zhengyi Liu ${ }^{1}$, Qiang Tang ${ }^{1}$, Xudan Zhang $^{1}$ and \\ Xiaogang Shu ${ }^{1}$
}

BACKGROUND: The miR-214 has been reported to be associated with various diseases, but its involvement in the pathophysiology of Hirschsprung disease (HSCR) is almost completely unexplored.

METHODS: In our study, we conducted a series of experiments to unravel the biological role of miR-214 in the pathophysiology of HSCR. qRT-PCR and western blotting were utilized to investigate the relative expression levels of miR-214, mRNAs, and proteins of related genes in colon tissues from 20 controls without HSCR and 24 patients with HSCR. The potential biological role of miR-214 in two cell lines (SKN-SH and SH-SY5Y) was assessed using the CCK8 assay, EdU staining, transwell assay, and flow cytometry. The dual-luciferase reporter assay was used to confirm PLAGL2 as a common target gene of miR-214.

RESULTS: All results suggested that miR-214 is upregulated in HSCR tissue samples compared with controls. Additionally, we found that miR-214 could inhibit cell proliferation and migration by directly downregulating the expression of PLAGL2, and the extent of the miR-214-mediated inhibitory effects could be rescued by a PLAGL2 overexpression plasmid.

CONCLUSION: Our results revealed that miR-214 is indeed involved in the pathophysiology of HSCR and suppresses cell proliferation and migration by directly downregulating PLAGL2 in cell models.

Pediatric Research (2019) 86:460-470; https://doi.org/10.1038/s41390-019-0324-9

\section{INTRODUCTION}

Hirschsprung disease (HSCR), known as aganglionosis and the most common neurocristopathy in humans, affects 1:2000-1:5000 newborns worldwide, ${ }^{1}$ and males are four times more affected compared to females. ${ }^{2}$ HSCR is a common congenital malformation of the gut, and it is mainly characterized by the fact that enteric neural crest cell (ENCCS) fail to invade, proliferate and migrate down the hindgut during embryonic development. ${ }^{3}$ This results in the deficiency of the ganglion cells in the submucosal and muscular layers along a variable portion of the distal gut. ${ }^{4}$ Among the clinical manifestations of HSCR, abdominal distension and delayed meconium excretion are common symptoms. ${ }^{5,6}$ The latest etiological studies have shown that the development of HSCR is a complicated process under the influence of both multiple genetic factors and environmental conditions. ${ }^{7,8}$ Particularly, genes correlated to the proliferation and migration of ENCCs play crucial parts in the pathogenesis of HSCR. ${ }^{9}$ To date, extensive research has identified more than 10 key genes, including RET, GDNF, and SOX10. ${ }^{10-12}$ Any factor involved in the proliferation, migration, invasion, and survival of ENCCs may be responsible for pathogenesis of the distal gut and thus could help cause HSCR development.

MicroRNAs (miRNAs) are an abundant class of non-coding and endogenous RNA molecules of 19-25 nucleotides, binding to the complementary $3^{\prime}$-untranslated regions ( $3^{\prime}$ UTR) of mRNAs and post-transcriptionally inhibit their expression either by destabilizing the target mRNAs or by interfering with translation. ${ }^{13,14}$ Currently, an increasing number of miRNAs have been reported in several biological processes, such as cell proliferation and migration. ${ }^{15}$ Recent studies have revealed that miR-214 participates in various diseases because of its influence on biological and pathological processes, such as ovarian cancer, ${ }^{16}$ gastrointestinal cancer, ${ }^{17}$ cervical cancer. ${ }^{18}$ The sum of the present literature reveals that miR-214, a molecular hub, participates in the control of the underlying biological networks of cancers and could be a promising diagnostic or prognostic biomarker and potential target for therapeutic intervention. ${ }^{19}$ However, whether and how miR-214 is involved in HSCR progression remains unclear, and its potential target gene also has not been completely determined. Thus, a better understanding of the biological role of miR-214 during ENCC development and human HSCR generation is necessary and urgent.

In our study, we carried out experiments to reveal the functional involvement of miR-214 and its interaction with its target gene in HSCR progression. We also have identified the target gene of miR214, the pleomorphic adenoma gene-like2 (PLAGL2), a transcription factor that has been shown to contribute to many vital biological processes, such as cell differentiation, apoptosis and proliferation. ${ }^{20-22}$ Therefore, to the best of our knowledge, we are

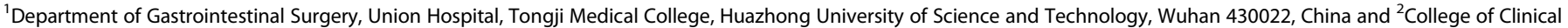
Medicine, Hubei University of Science and Technology, Xianning 437100, China

Correspondence: Xiaogang Shu (sxg678@yahoo.com)

${ }^{3}$ Present address: Department of Gastrointestinal Surgery II, Renmin Hospital of Wuhan University, Wuhan 430060, China

These authors contributed equally: Liang Wu, Wenzheng Yuan

Received: 10 February 2018 Revised: 4 December 2018 Accepted: 7 December 2018

Published online: 1 March 2019 
the first group to investigate the detailed mechanism of the miR214-PLAGL2 interaction in the pathogenesis of HSCR.

\section{MATERIALS AND METHODS}

Patient specimens

All of the experiments in our study were carried out in accordance with the Helsinki Declaration, and approved by the Ethics Committee Tongji Medical College, Huazhong University of Science and Technology (IORG0003571). Our research utilized 44 colon tissue samples, including 24 HSCR patient samples and 20 matched controls. Both HSCR and control group specimens were collected from patients at the Department of Pediatric Surgery at Union Hospital of Tongji Medical College with full understanding and consent obtained from their guardians. All fresh samples gathered were immediately frozen after operative treatment and then stored at $-80{ }^{\circ} \mathrm{C}$ until use. The traditional anorectal manometry and barium enema evaluation before surgery were used extensively to diagnose all HSCR patients enrolled in this study, and pathological analysis for definitive diagnosis was performed after surgery. A total of 20 matched control specimens, without the ischemia or necrosis parts and verified without HSCR, were collected from patients who underwent surgical intervention as a result of intussusception and inguinal hernia.

RNA isolation and qRT-PCR analysis

The total RNAs containing miRNAs were isolated from $24 \mathrm{HSCR}$ patient samples, 20 matched controls and two cell lines using RNAiso Plus (Takara, Tokyo, Japan) as described by the manufacturer. Then, qPCR was performed to detect the relative expression levels of miR-214 and all related mRNAs. To assess the mRNA and miR-214 expression levels, a reverse transcription kit (Takara) and TaqMan microRNA assays (RiboBio, Guangzhou, China) were applied in these experiments. GAPDH and U6 were used to normalize the level of the mRNAs and miRNA, respectively. The primers using in RT-PCR are shown in Table 1.

Western blot

The total proteins were extracted from human colon tissues and cultured cells for western blotting analysis, mixed with $1 \times$ cell lysis buffer containing protease inhibitors (Beyotime, Wuhan, China). Equal amount of denatured proteins separated with 10\% SDS-PAGE (Servicebio, Wuhan, China), were transferred to polyvinylidene fluoride (PVDF) membranes (Servicebio). The PVDF membranes with the target proteins were blocked with $5 \%$ skimmed milk (Servicebio) and incubated with various primary antibodies (1:1000, Proteintech, Wuhan, China) at $4{ }^{\circ} \mathrm{C}$ overnight. After rinsing, the

\begin{tabular}{|lll|}
\hline \multicolumn{2}{l}{ Table 1. } & Primer sets used for \\
\hline Primer set & Primers & Sequence $\left(5^{\prime}-3^{\prime}\right)$ \\
\hline GAPDH & Forward & CATGTTCGTCATGGGGTGAACCA \\
& Reverse & AGTGATGGCATGGACTGTGGTCAT \\
PLAGL2 & Forward & GAGGCCCTCAGCTCAGTTTT \\
& Reverse & CAAATGGTTCAGGCTGGTGC \\
ITCH & Forward & CGCTCTAGTAGCTGTGGTCG \\
& Reverse & ACATGGCAAAGGAGACACTGA \\
PTEN & Forward & TGTAGTAAGTTGTGCTGAAAGACA \\
& Reverse & CGGCTGAGGGAACTCAAAGT \\
U6 & Forward & AAAGCAAATCATCGGACGACC \\
& Reverse & GTACAACACATTGTTTCCTCGGA \\
miR-214 & Forward & GATGAGCTCAACTGAAGTGGCTAAAGAG \\
& Reverse & GATACGCGTTGAAGTTCTGCCTAATCTA \\
\hline
\end{tabular}

PVDF membranes were further incubated with the appropriate secondary antibody (Servicebio) for $1 \mathrm{~h}$ at room temperature. An ECL reagent (Servicebio) was employed to visualized band signals, and the band densities were detected and quantified by using the ImageJ software.

Cell culture and transfection

As a result of the limitation of the enteric nervous system (ENS) cell model of HSCR, according to previous reporting papers, two most felicitous cell lines, human SKN-SH and SH-SY5Y cells, are neuroblastoma cell lines which derived from NCCs and used to investigate the mechanism of HSCR in many articles. ${ }^{23-25}$ These two cell lines were obtained from Dr. Qiangsong Tong, at Department of Pediatric Surgery, Union Hospital, Tongji Medical College, Huazhong University of Science and Technology, China. ${ }^{26}$ These two cell lines were used via in vitro experiments and cultivated in a humidified atmosphere of $5 \% \mathrm{CO}_{2}$, at $37{ }^{\circ} \mathrm{C}$, with DMEM containing high glucose levels (Boster, Wuhan, China), 10\% fetal bovine serum (FBS) (Sciencell, Beijing, China), streptomycin $(100 \mu \mathrm{g} / \mathrm{mL}$ ) and penicillin $(100 \mathrm{U} / \mathrm{mL}$ ) (SigmaAldrich, St. Louis, MO). In the transfection experiments, synthetic miR-214 mimics, a miR-214 inhibitor(2,O-methylmodification) (the sequences, shown in Table 2), PLAGL2 siRNA (Ribobio), the overexpression plasmid of the PLAGL2 gene (Genecopoeia, Guangzhou, China) and the respective negative controls were used with the RiboBio FECT ${ }^{\mathrm{TM}} \mathrm{CP}$ Transfection Kit according to the manufacturer's protocols.

Cell cycle and apoptosis analysis

To estimate the biological role of miR-214 in the cell cycle distribution and apoptosis, we transfected SKN-SH and SH-SY5Y cell with miR-214 mimics and negative controls for $48 \mathrm{~h}$, the transfected cells in log-phase of growth were gathered and analyzed with a BD FACSFlow Cytometer (BD Biosciences, Franklin Lakes, NJ). For detecting cell apoptosis, Annexin V-FITC and PI (Antgene, Wuhan, China) were applied for $30 \mathrm{~min}$ at $37^{\circ} \mathrm{C}$ to stain these harvested cells. Additionally, transfected cells were harvested for cell cycle analysis, washed with cold PBS, and fixed with $75 \%$ cold ethanol. Before analysis with a BD FACSFlow Cytometer, the cells were incubated with PI $(50 \mu \mathrm{g} / \mathrm{mL}$, AntGent) for $10 \mathrm{~min}$.

Cell proliferation

The cell proliferation after 24,48 , and $72 \mathrm{~h}$ of transfection was evaluated using a CCK8 assay (Dojindo, Tokyo, Japan). Transfected and control cells $\left(3 \times 10^{3}\right.$ cells $\left./ 100 \mu \mathrm{L}\right)$ were seeded into a 96-well plate. CCK8 reagent was added to each well, and then, the samples were subjected to a $2 \mathrm{~h}$ incubation at $37^{\circ} \mathrm{C}$. The resulting absorbance at $450 \mathrm{~nm}$ was detected for each sample (Tecan, Mechelen, Belgium). To further detect cell proliferation, the EdU Cell Proliferation Assay Kit (Ribobio) was also applied. After $48 \mathrm{~h}$ transfection, SH-SY5Y and SKN$\mathrm{SH}$ cells seeded in 96 -well plates. Briefly, the cells were incubated with $50 \mu \mathrm{M}$ EdU at $37^{\circ} \mathrm{C}$ for $2 \mathrm{~h}$ before fixation, permeabilization and EdU staining, performed according to the manufacturer's protocol.

Table 2. Sequence of miR-214 mimics, a miR-214 inhibitor and the negative controls used in this study

\begin{tabular}{ll}
\hline miRNA & Sequence $\left(5^{\prime}-3^{\prime}\right)$ \\
\hline miR-214 mimics & ACAGCAGGCACAGACAGGCAGU \\
Mimic NC & ACUGCCUGUCUGUGCCUGCUGU \\
& UUUGUACUACACAAAAGUACUG \\
miR-214 inhibitor & CAGUACUUUUGUGUAGUACAAA \\
Inhibitor NC & ACUGCCUGUCUGUGCCUGCUGU \\
\end{tabular}


Nuclei were counterstained with Hoechst 33342 (Ribobio) at room temperature for $30 \mathrm{~min}$.

Cell transwell assays

To detect the capacity for cell migration, transwell migration chambers ( $8 \mu \mathrm{m}$ pore size, Corning-Costar, Shanghai, China) were used. Both cell lines were transfected with miR-214 mimics, PLAGL2 siRNA, a PLAGL2 overexpression plasmid and negative controls. After transfection for $48 \mathrm{~h}$, the cells were resuspended as a single-cell suspension $\left(1 \times 10^{6}\right.$ cells $\left./ \mathrm{ml}\right)$ with FBS-free medium. Then, $100 \mu \mathrm{l}$ of the suspension was seeded into the upper chamber, and $600 \mu$ DMEM with $10 \%$ FBS was placed in the lower chamber as the chemotaxin. After $24 \mathrm{~h}$ of incubation, the cells were fixed with a $4 \%$ paraformaldehyde solution (Servicebio) for $20 \mathrm{~min}$ and stained with a crystal violet staining solution (Servicebio) for $30 \mathrm{~min}$. The migrated cells on the bottom of the membranes were visualized under $\times 200$ magnification and counted (six random views per well) with the ImageJ software.

Bioinformatics prediction of the target gene for miR-214

Three main databases (DIANA LAB (http://diana.imis.athenainnovation.gr/DianaTools/index.php), Targetscan (http://www. targetscan.org/), and Pictar (http://pictar.mdc-berlin.de/)) were used to predict the potential target genes regulated by miR-214. ${ }^{27-29}$ Additionally, gene function analyses were also considered.

Immunohistochemical analysis

The removed HSCR and control group specimens were fixed in 4\% paraformaldehyde followed by embedding in paraffin. Sections $4 \mu \mathrm{m}$ thick were stained with hematoxylin-eosin (HE) staining and immunofluorescence staining. For immunofluorescence staining, the primary rabbit anti-PLAGL2 (1:50 dilution, Proteintech) and mouse anti-Calretinin antibody (1:50 dilution, Proteintech) were applied to the sections and the slides were incubated overnight at $4{ }^{\circ} \mathrm{C}$. Then, the sections were washed and reacted with the secondary antibodies Alexa Fluor 594 donkey-anti mouse (1:200 dilution, Invitrogen, Paisley, UK) to detect Calretinin, and Alexa Fluor 488 donkey-anti rabbit (1:200 dilution, Invitrogen), to detect PLAGL2, for $2 \mathrm{~h}$ at room temperature. Nuclei were counterstained with DAPI (Invitrogen).

\section{Dual-luciferase reporter assay}

To verify whether miR-214 directly regulates PLAGL2 by combining the $3^{\prime}$ UTR region of PLAGL2 mRNA, a dual-luciferase reporter assay (Promega, Madison, WI) was used. There are three potential binding sites in the $3^{\prime}$ UTR region of PLAGL2 mRNA. Thus, wild-type and mutated $3^{\prime}$ UTR sequence of PLAGL2 mRNA, called pGL3-PLAGL2wild1-3 and pGL3-PLAGL2-mut1-3, respectively, were inserted between the hRluc and the hLuc genes in the pGL3 promoter vector. According to the manufacturers' protocols, SH-SY5Y cells were seeded in 96-well plates and then co-transfected with $100 \mathrm{ng}$ of pGL3-PLAGL2-wild1-3/pGL3-PLAGL2-mut1-3 and $50 \mathrm{nM}$ miR214 mimics, the miR-214 inhibitor or the respective negative controls, using the RiboBio FECT ${ }^{\mathrm{TM}}$ CP Transfection Kit (RiboBio). The values of Renilla/firefly luciferase activities in the cell lysates were ultimately used for analysis.

Statistical analysis

All results shown in our research were independently repeated at least three times and analyzed using SPSS22 statistical software. They were presented using GraphPad. The differential expression of miR-214 between 24 HSCR patient samples and 20 matched controls is shown as a box and whisker graph. The data obtained from the in vitro experiments were presented as the mean \pm standard error (SE). A double-sided Student's $t$-test was applied to analyze these data. The correlation between the expression levels of miR-214 and PLAGL2 in the case and control groups was estimated using Pearson's correlation. Differences in mean values of $p<0.05$ were considered statistically significant.

\section{RESULTS}

Patient samples information analysis

In total, 24 HSCR-confirmed specimens and 20 matched controls were gathered in our study. Patient samples information, age and gender were collected. The age of the HSCR patients and the matched controls were $5.704 \pm 0.761$ and $6.255 \pm 0.8863$ months old, respectively, which shown no statistically significant differences. The gender rates (male/female) among HSCR patients and the matched controls were $17: 7$ and 12:8, respectively, which is generally consistent with the average gender rate of HSCR in human beings. The golden criterion for diagnosing $H D$ is the absence of ganglion cells in the myenteric or submucosal nerve plexus of the intestine and the presence of hypertrophic nerve trunks in the submucosa. To assess histopathology of HSCR, the immunohistochemical staining for hematoxylin and eosin (HE), calretinin and S100 was performed on HSCR and control group specimens. ${ }^{30}$ In control group specimens, calretinin staining for ganglion cells is positive and S100 staining highlighted negatively stained ganglion cells as a cell-shaped blank area. No calretinin staining was observed in HSCR tissues. Dense and prominent S100 immunostaining showed hypertrophic nerve trunks in HSCR specimens (Fig. 1a).

Overexpression of miR-214 in HSCR

The results shown in our study revealed that the level of miR-214 expressed in HSCR specimens was upregulated compared with that in the controls ( $P=0.0017$, Fig. $1 \mathrm{~b})$, indicating miR-214 might be associated with the pathogenesis of HSCR.

The miR-214 mimics inhibited the cell proliferation and migration Because miR-214 is upregulated in the HSCR-confirmed tissues, we first investigated its functional roles in cell proliferation and migration by overexpressing miR-214. To achieve this goal, miR-214 mimics and a negative control (Fig. 1c) were transfected into human SKN-SH and SH-SY5Y cell lines, which then were subjected to a CCK8 assay, EdU staining and a transwell assay. The miR-214 mimics could remarkably suppress the ability of the proliferation and migration of two cell lines compared with that of the matched group, which suggests that the overexpression of miR-214 has a significant inhibitory effect on cell proliferation and migration (Fig. 1d-f).

The miR-214 mimics did not impact apoptosis or cell cycle Flow cytometry analysis was performed to estimate the biological function of miR-214 in cell cycle and apoptosis. These results indicated that both the percentage of apoptotic cells and the cell distribution in the cell cycle all have no significant differences between the cells transfected with miR-214 mimics and matched controls (Fig. 2a-d).

PLAGL2 was a common target gene of miR-214

PLAGL2 has been shown to regulate many vital biological processes, such as cell differentiation, proliferation, and apoptosis. We have examined accidentally before that PLAGL2 expression was prominently down-regulated in HSCR samples compared to the matched controls. ITCH and PTEN, were more frequently reported to be regulated by miR-214. So three potential target genes, PLAGL2, ITCH, and PTEN, were selected. To examine whether all three potential target genes were related to the pathogenesis of HSCR, we applied western blot to detect the protein expression level of related genes between the 24 HSCR samples and 20 matched controls. PLAGL2 expression was prominently down-regulated in HSCR samples compared to the matched controls $(P<0.001$, Fig. 3a, b). In contrast, the expression levels of ITCH and PTEN did not vary observably 
a

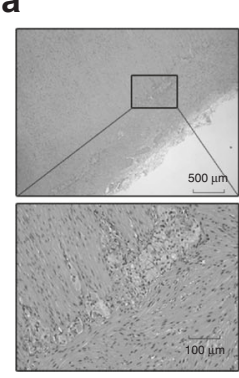

HE
Control

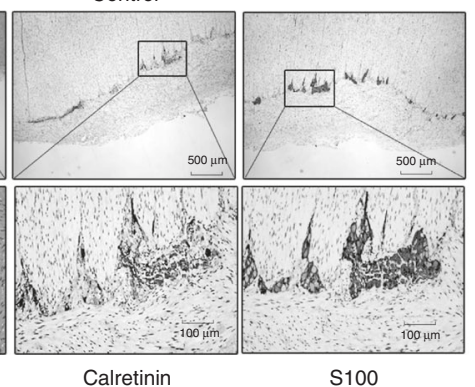

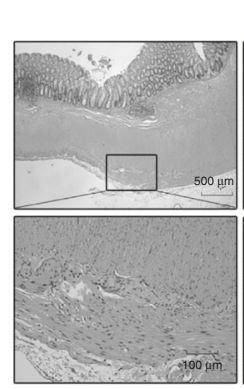

HE
HSCR

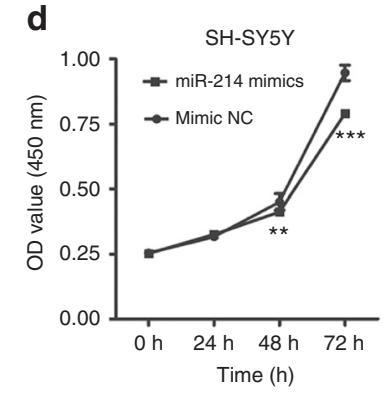

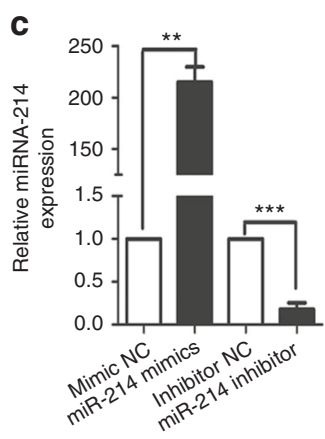

b

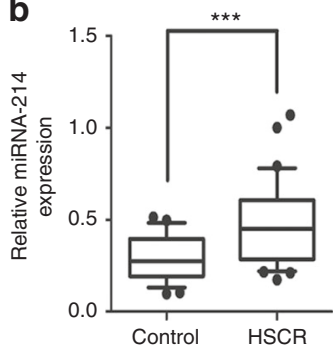

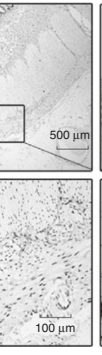

alretinin
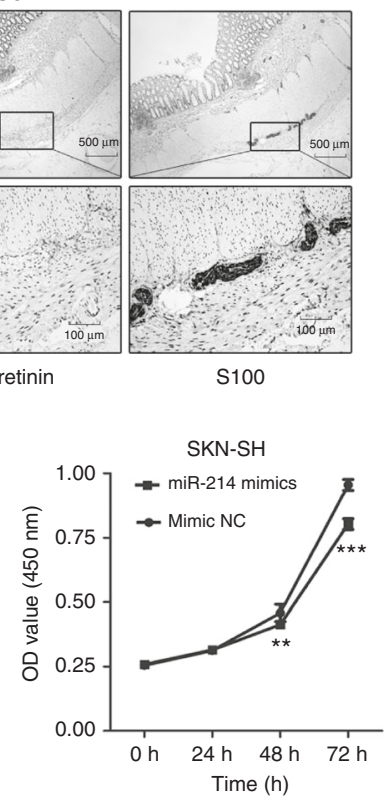

e
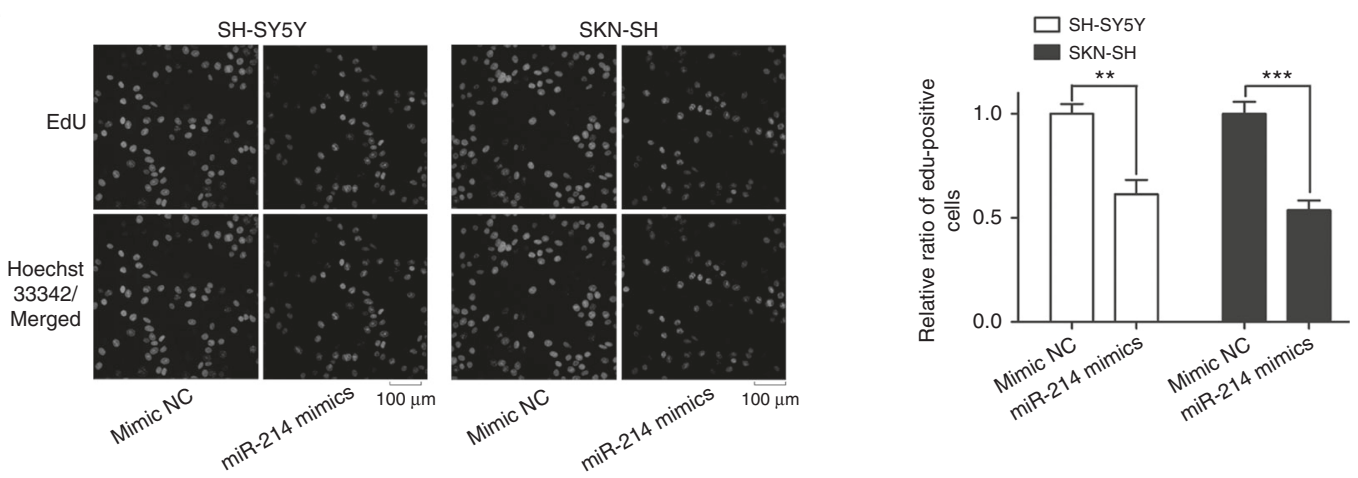

f
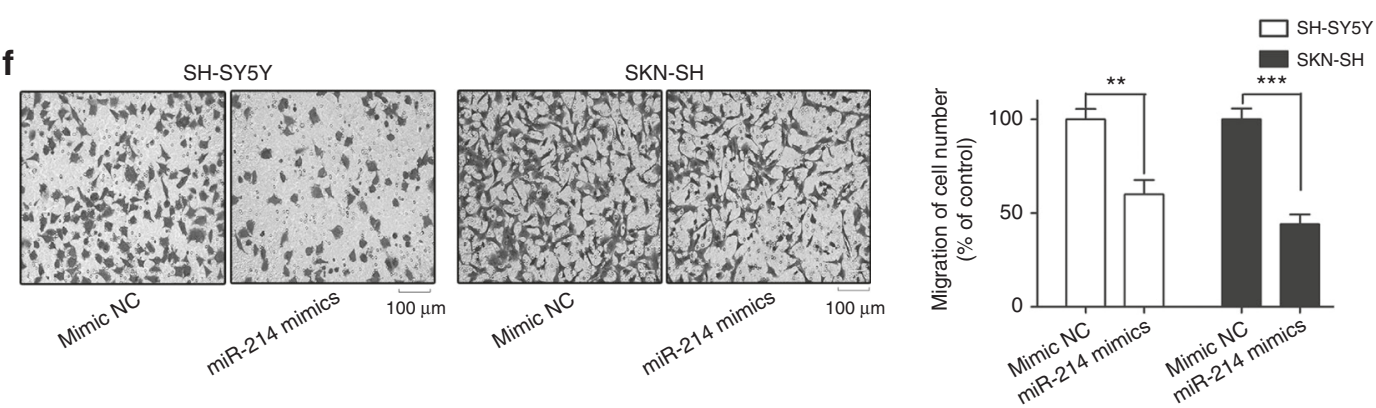

Fig. 1 The overexpression of miR-214 in HSCR samples and the effects of the miR-214 mimics on cell growth and migration. * indicates a significant difference compared with control group $(P<0.05)$. ** indicates significant diferrences $(P<0.01)$ and $* * *$ indicates significant diferrences $(P<0.001)$. a the immunohistochemical staining for hematoxylin and eosin (HE), calretinin and S100 in HSCR and control group specimens. In control group specimens, calretinin staining for ganglion cells is positive and S100 staining highlighted negatively stained ganglion cells as a cell-shaped blank area. No calretinin staining was observed in HSCR tissues. Dense and prominent S100 immunostaining showed hypertrophic nerve trunks in HSCR specimens. b The expression of miR-214 in 24 HSCR cases was significantly higher compared to 20 matched controls $(P=0.0017)$. The expression level of miR-214 was analyzed using qRT-PCR and normalized with the data for U6. The data are presented as a box plot of the median and range of the log-transformed relative expression levels. $c$ The relative expression level of miR-214 in the cells transfected with miR-214 mimics, miR-214 inhibitor, and the negative controls. d-f The effects of the miR-214 mimics on cell growth and migration. Representative images and the quantifications of migrated cells are shown. EdU proliferation assay analysis of the effect of miR-214 on the growth of SH-SY5Y and SKN-SH cells. The red fluorescent cells are in the S phase of mitosis, and the blue fluorescent cells represent all of the cells. The absorbance at $450 \mathrm{~nm}$ was presented as the mean $\pm \mathrm{SE}$. The migrated cells on the bottom of the membranes were visualized under $200 \times$ magnification and counted (six random views per well) with the ImageJ software 

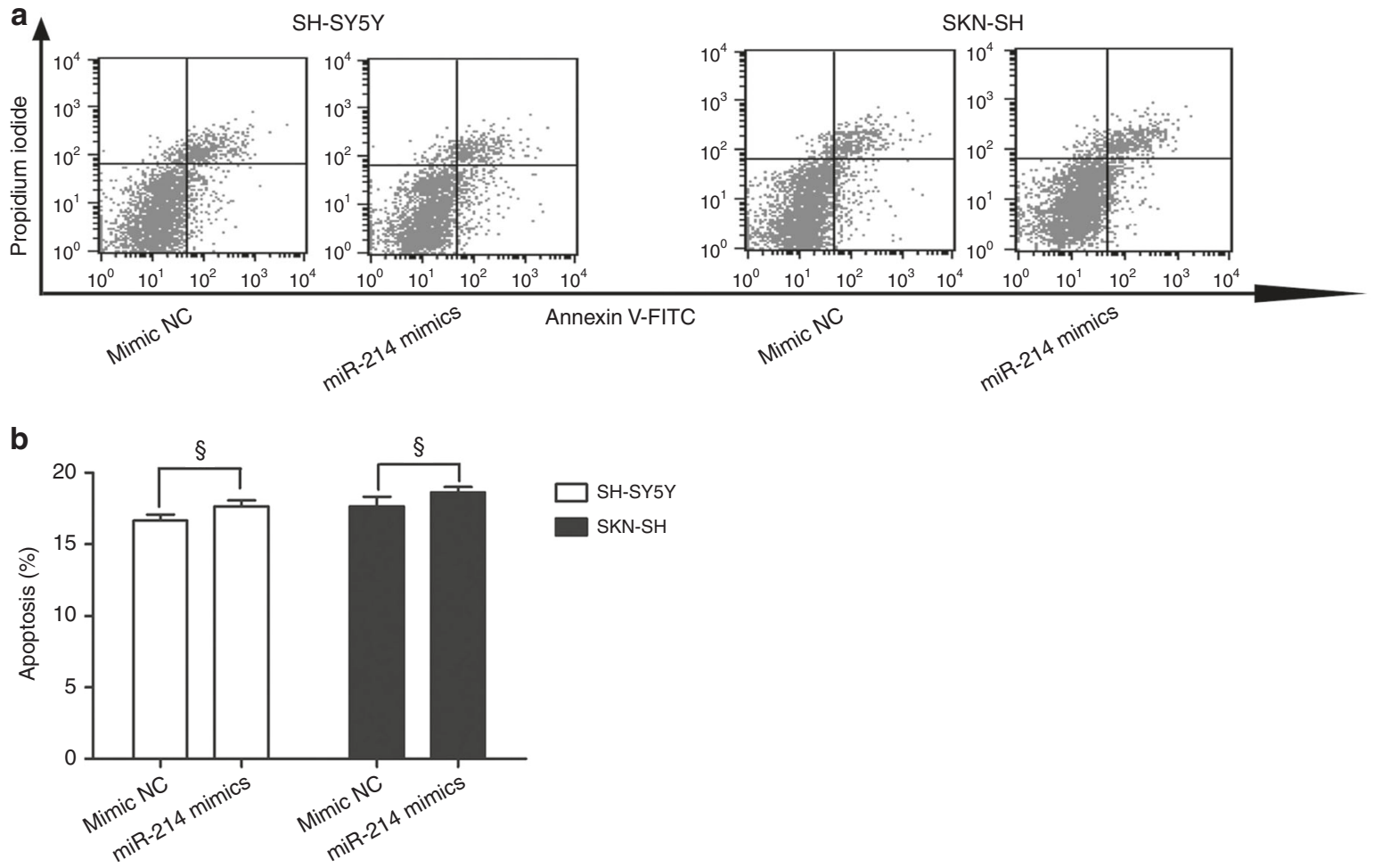

C SH-SY5Y
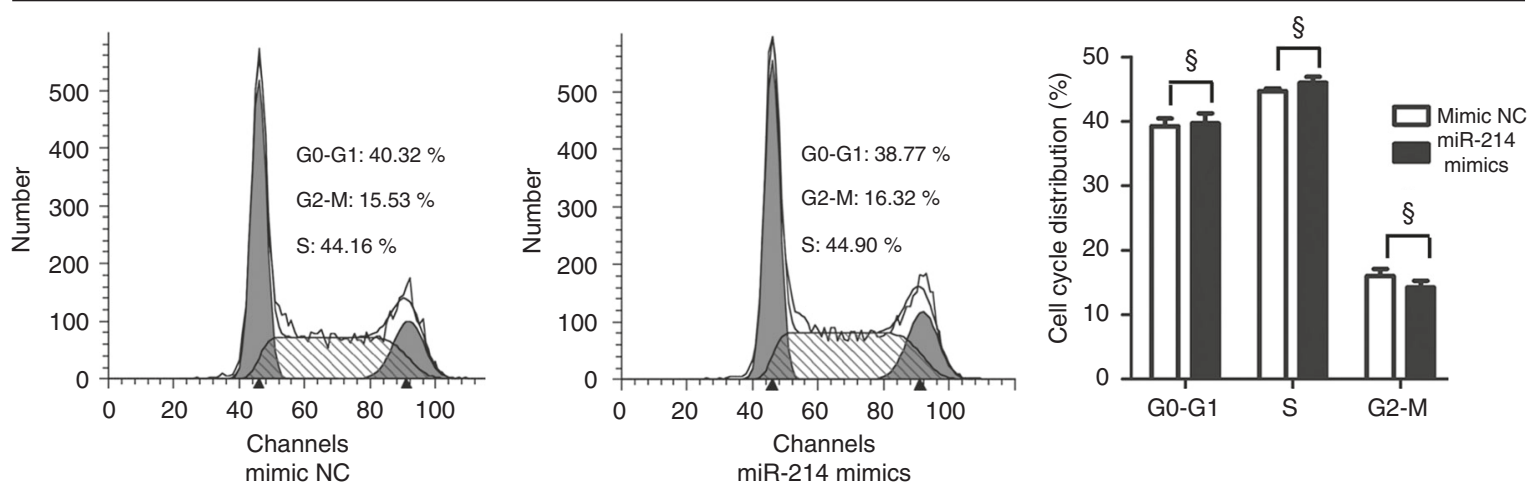

d
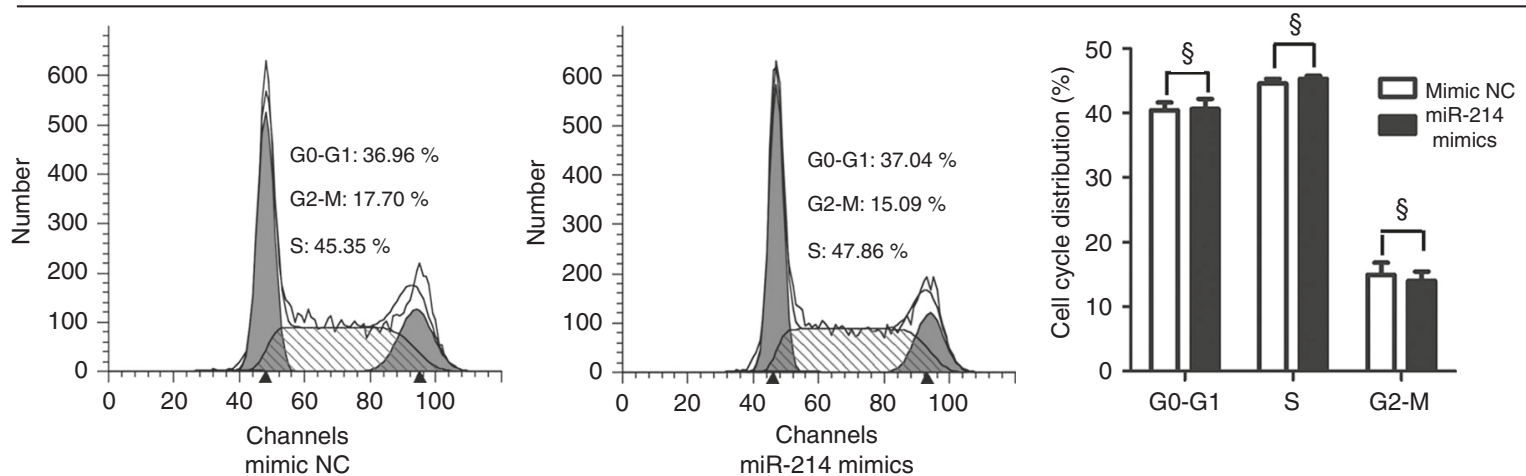

Fig. 2 The effect of miR-214 mimics on apoptosis and cell cycle. $\S$ indicates no significant diferrence. a-d A flow cytometry assay was performed to evaluate the effect of miR-214 mimics on apoptosis and cell cycle

(Fig. 3a, b). Immunofluorescence analysis of PLAGL2 (Alexa Fluor 488, green) and Calretinin (Alexa Fluor 594, red) shows that PLAGL2 was mainly expressed in lamina propria and the areas surrounding myenteric plexuses of control group specimens
(Fig. 3c). The distribution of PLAGL2 in HSCR samples has no significant difference (Fig. $3 \mathrm{C}$ ). We have identified three putative miR-214 binding sites at position 442-448, 1461-1467, and 3430-3436(CCTGCTG) in $3^{\prime}$-UTR of PLAGL2 mRNA using 
a

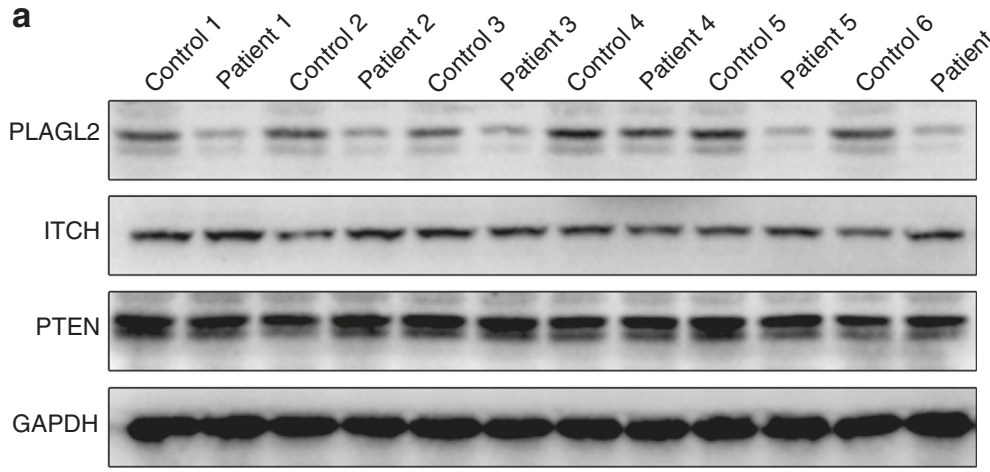

C PLAGL2/Calretinin/DAPI

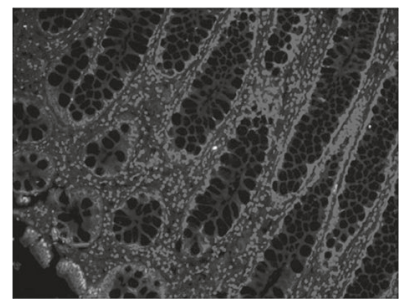

Mucosae and submucosa

Control

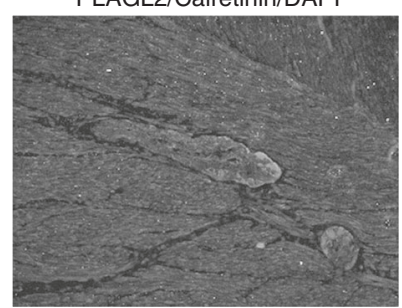

Myenteric plexus b

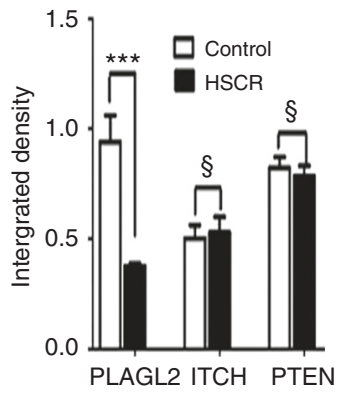

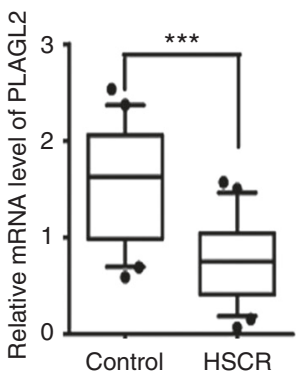

HSCR d

\begin{tabular}{|c|c|}
\hline $\begin{array}{l}\text { PLAGL2 3' UTR Wild Type } 1 \\
\text { hsa-miR-214-3p }\end{array}$ & $\begin{array}{c}5^{\prime} \quad \ldots \text { GUCAGUUGGUCAGGCCCUGCUGU...3' } \\
\text { UGACGGACAGACACGGACGACA }\end{array}$ \\
\hline PLAGL2 3' UTR Mutant 1 & $\begin{array}{lll}5^{\prime} & \ldots \text { GUCAGUUGGUCAGGC }\end{array}$ \\
\hline $\begin{array}{l}\text { PLAGL2 3' UTR Wild Type } 2 \\
\text { hsa-miR-214-3p } \\
\text { PLAGL2 3' UTR Mutant } 2\end{array}$ & $\begin{array}{c}5^{\prime} \ldots \text { UGACUCGUGGGGCUCCCUGCUGC...3' } \\
\text { UGACGGACAGACACGGACGACA } \\
5^{\prime} \text {...UGACUCGUGGGGCUCGGACGAC...3' }\end{array}$ \\
\hline $\begin{array}{l}\text { PLAGL2 3' UTR Wild Type } 3 \\
\text { hsa-miR-214-3p } \\
\text { PLAGL2 3' UTR Mutant } 3\end{array}$ & $\begin{array}{c}5^{\prime} \ldots \text { UCCUGGAACUCUGAACCUGCUGU....3' } \\
\text { UGACGGACAGACACGGACGACA } \\
5^{\prime} \text {...UCCUGGAACUCUGAAGGACGACU...3 }\end{array}$ \\
\hline
\end{tabular}

PLAGL2/Calretinin/DAPI

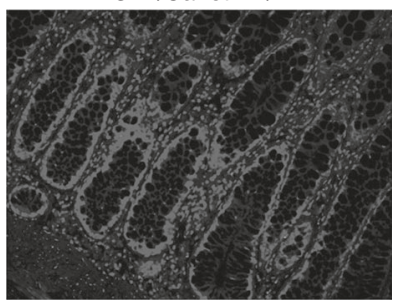

Mucosae and submucosa
PLAGL2/Calretinin/DAPI

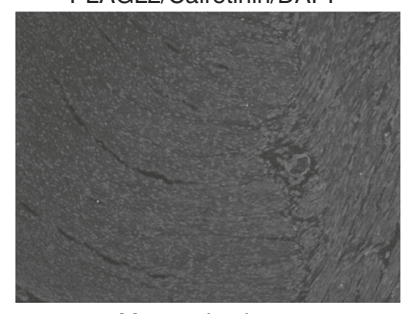

Myenteric plexus

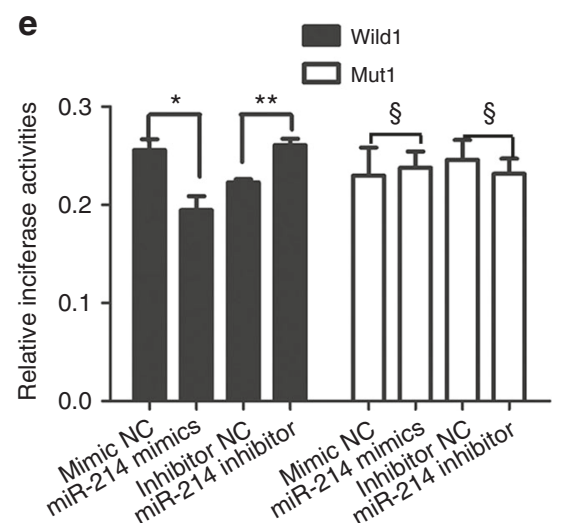

f

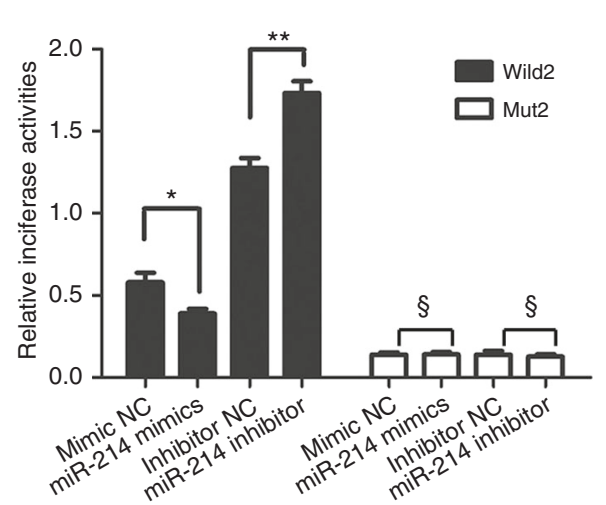

g

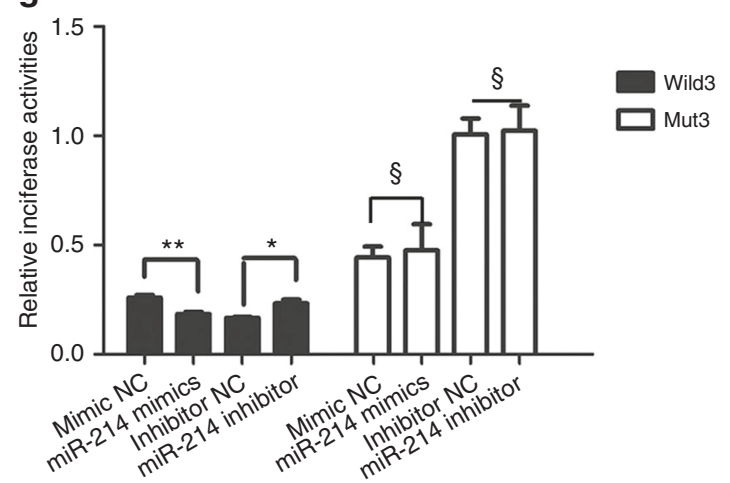

Fig. 3 PLAGL2 was a common target gene of miR-214. a, b The protein and RNA expression levels of PLAGL2, ITCH, and PTEN in human HSCR case and control tissues. c Immunofluorescence analysis of the PLAGL2 expression distribution. PLAGL2 (Alexa Fluor 488, green), Calretinin (Alexa Fluor 594, red). Nuclei were counterstained with DAPI. PLAGL2 was mainly expressed in lamina propria and the areas surrounding myenteric plexuses of control group specimens. The distribution of PLAGL2 in HSCR samples has no significant difference. d The sequence alignment of human miR-214 with the $3^{\prime}$ UTR of PLAGL2. There are three potential binding sites in the $3^{\prime}$ UTR region of PLAGL2 mRNA, The wild-type and mutated $3^{\prime}$ UTR sequences of PLAGL2 mRNA were called pGL3-PLAGL2-wild1-3 and pGL3-PLAGL2-mut1-3, respectively. e-g The co-transfection of miR-214 mimics with pGL3-PLAGL2-wild1-3 remarkably suppressed the luciferase activity in the human SH-SY5Y cell line compared with the negative controls. However, no significant alteration between the luciferase activity of pGL3-PLAGL2-mut1-3 and negative controls was detected 
TargetScan analysis (Fig. 3d). To further confirm the direct binding of miR-214 to the $3^{\prime}$-UTR of PLAGL2 mRNA, the dualluciferase reporter assay was applied. There are three potential binding sites in the $3^{\prime}$ UTR region of PLAGL2 mRNA. The cotransfection of miR-214 mimics with pGL3-PLAGL2-wild remarkably suppressed the luciferase activity in the human SH-SY5Y

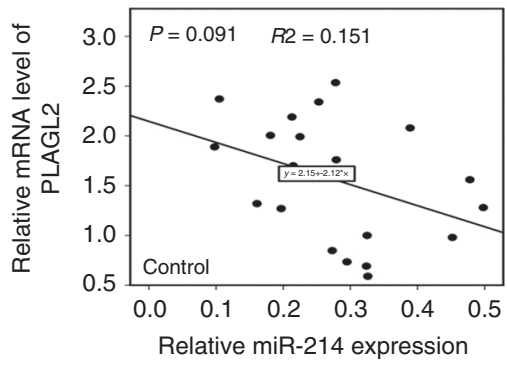

PLAGL2

GAPDH
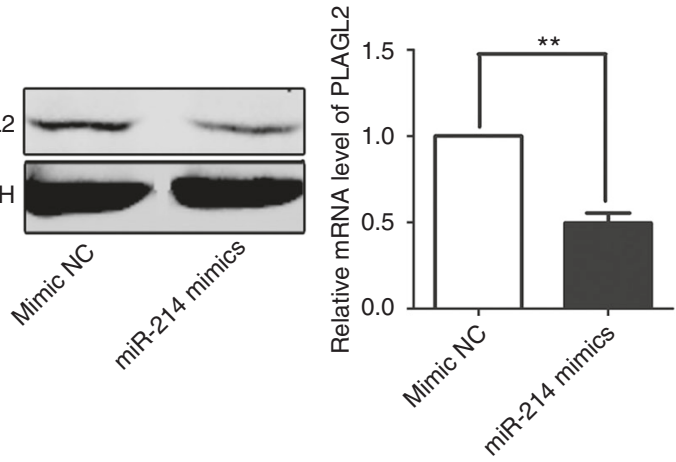

cell line compared with the negative controls. However, no significant alteration between the luciferase activity of pGL3PLAGL2-mut1-3 and negative controls has been detected. In contrast, the co-transfection of miR-214 inhibitors with pGL3PLAGL2-wild1-3 notably increased the luciferase activity compared with the controls (Fig. $3 \mathrm{e}-\mathrm{g}$ ).

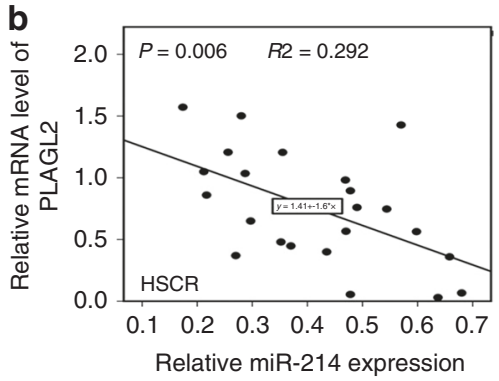

SH-SY5Y

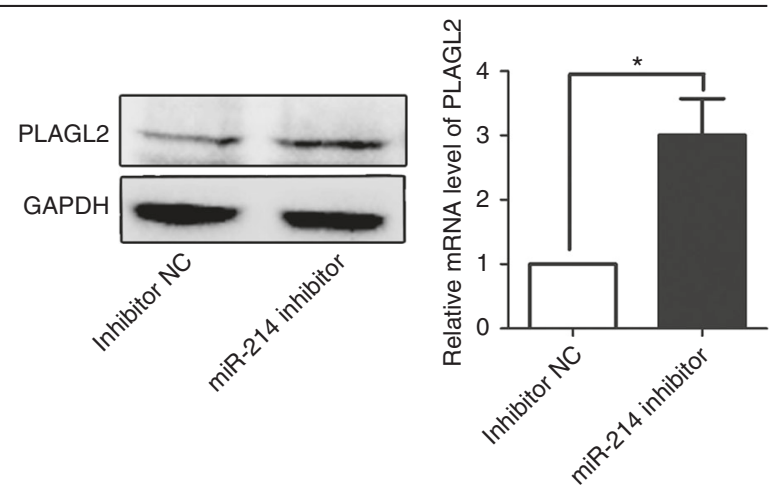

d

SKN-SH
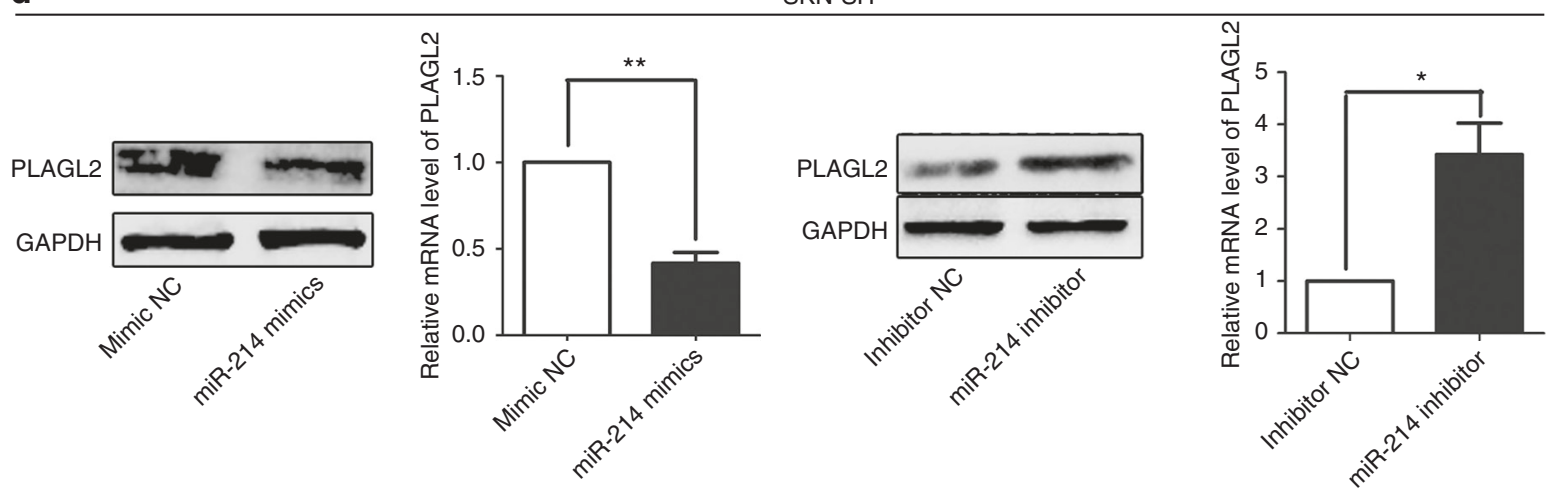

e
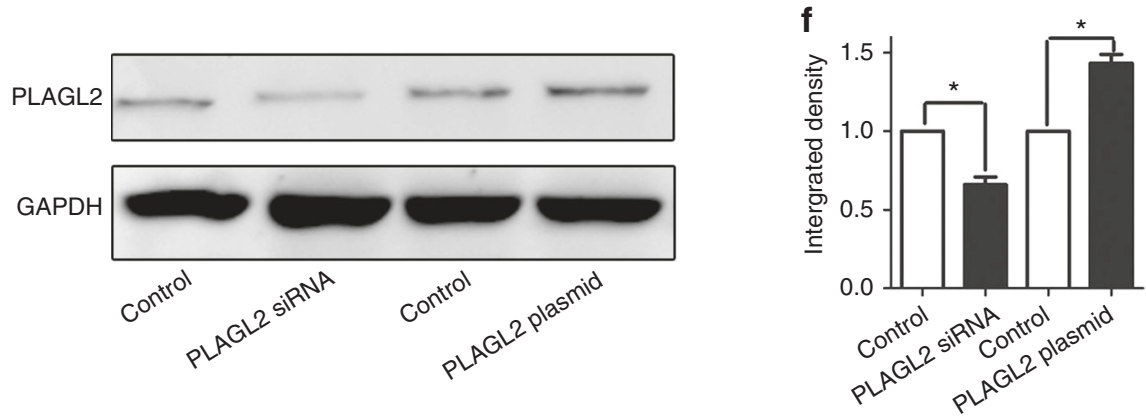

Fig. 4 The mRNA and protein expression levels of PLAGL2 were regulated by the miR-214. $\mathbf{a}$, $\mathbf{b}$ The correlation was examined in HSCR cases, and it showed intense relationships between miR-214 and PLAGL2 $\left(P=0.006, R^{2}=0.292\right)$, while a poor correlation was shown for the controls $(P \geq 0.05)$. c, d Two cell lines were transfected with $100 \mathrm{nM}$ of miR-214 mimics, a miR-214 inhibitor and the respective controls for $48 \mathrm{~h}$. Then, qRT-PCR and western blotting were performed to determine the mRNA and protein levels of PLAGL2. e, $\mathbf{f}$ The protein expression levels of PLAGL2 in the cells transfected with PLAGL2 siRNA, PLAGL2 plasmid, and the negative controls 
In summary, these experimental results strongly indicate that PLAGL2 is directly targeted and inhibited by miR-214 in HSCR.

Correlation between the miR-214 and PLAGL2 expression levels The correlation between the miR-214 and PLAGL2 relative expression levels between HSCR specimens and controls was estimated by Pearson's correlation. The analysis revealed that a distinct inverse correlation was observed between the levels of miR-214 and PLAGL2 in HSCR tissues $\left(P=0.006, R^{2}=0.292\right)$ but not in the matched controls $\left(P=0.091, R^{2}=0.151\right.$, Fig. $\left.4 \mathrm{a}, \mathrm{b}\right)$.
The miR-214 mimics downregulated PLAGL2 expression

To experimentally confirm PLAGL2, a direct target of miR-214, the two cell lines were transfected with miR-214 mimics, miR-214 inhibitors, and the respective negative controls. After $48 \mathrm{~h}$ of transfection, we respectively utilized qRT-PCR and western blotting to assess the mRNA and protein expression levels of PLAGL2. As expected, both the PLAGL2 mRNA and protein levels were significantly downregulated by the miR-214 mimics and upregulated by the miR-214 inhibitor in two cell lines (Fig. 4c, d). a

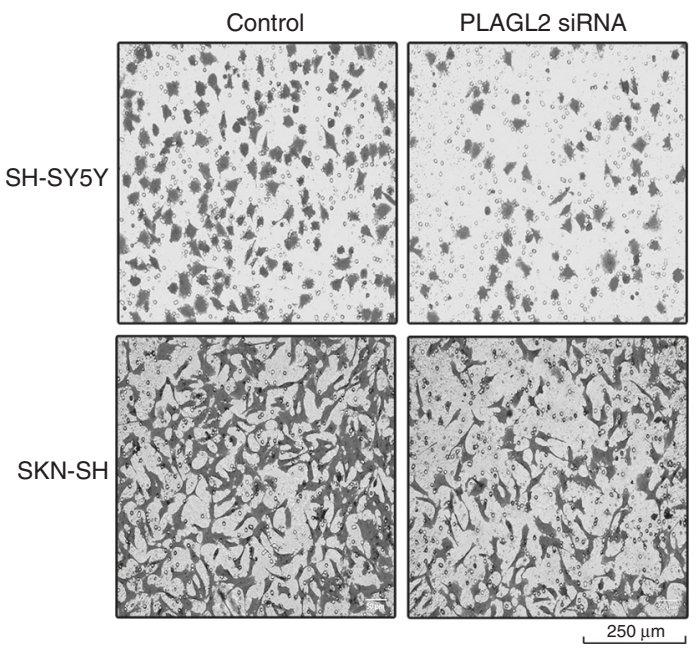

b

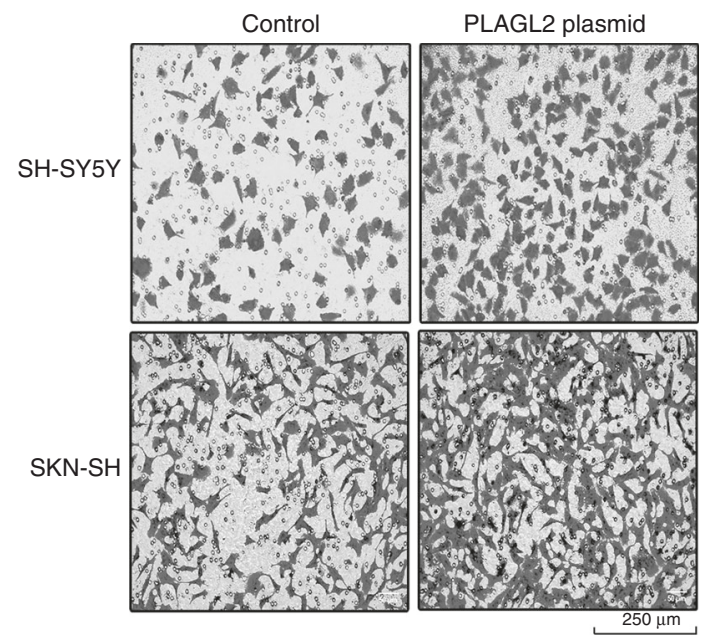

c

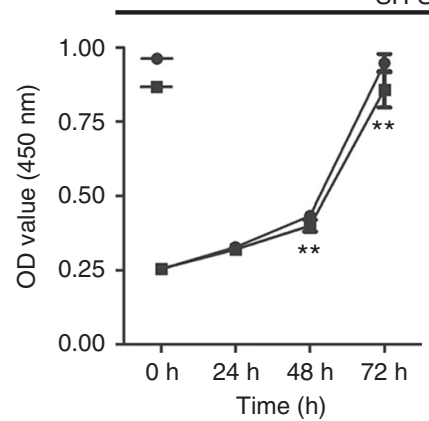

SH-SY5Y

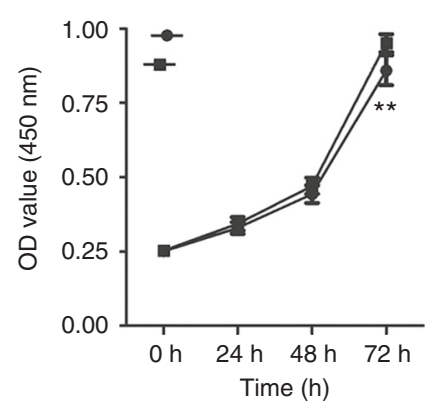

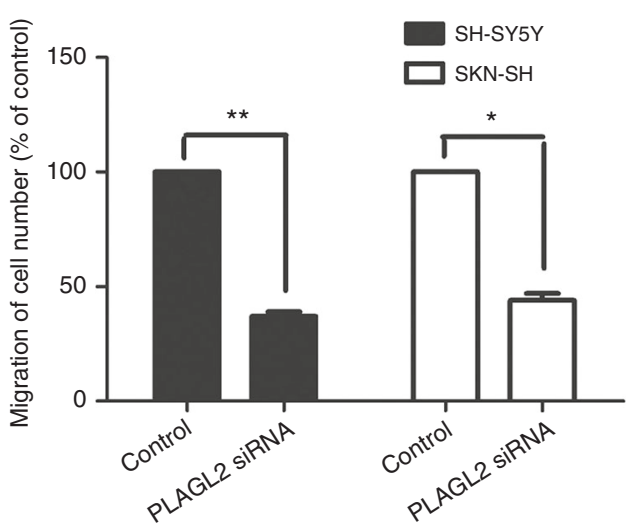

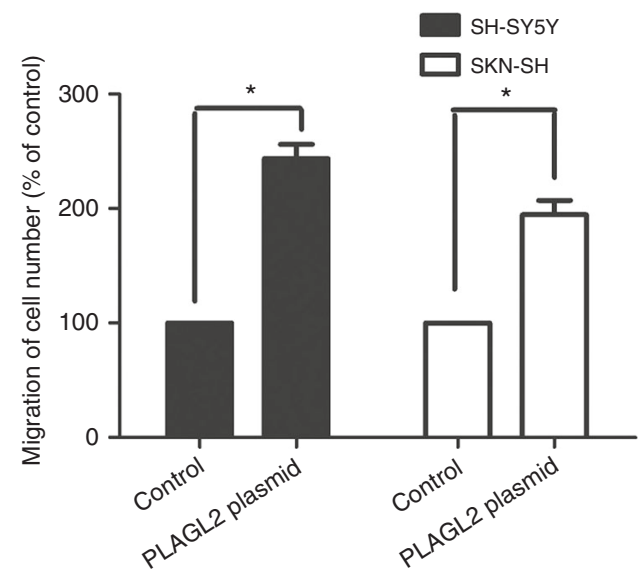

d

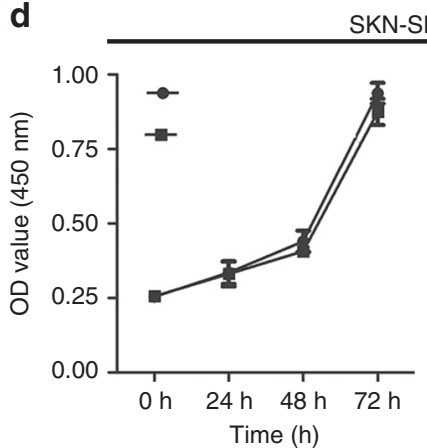

.


a

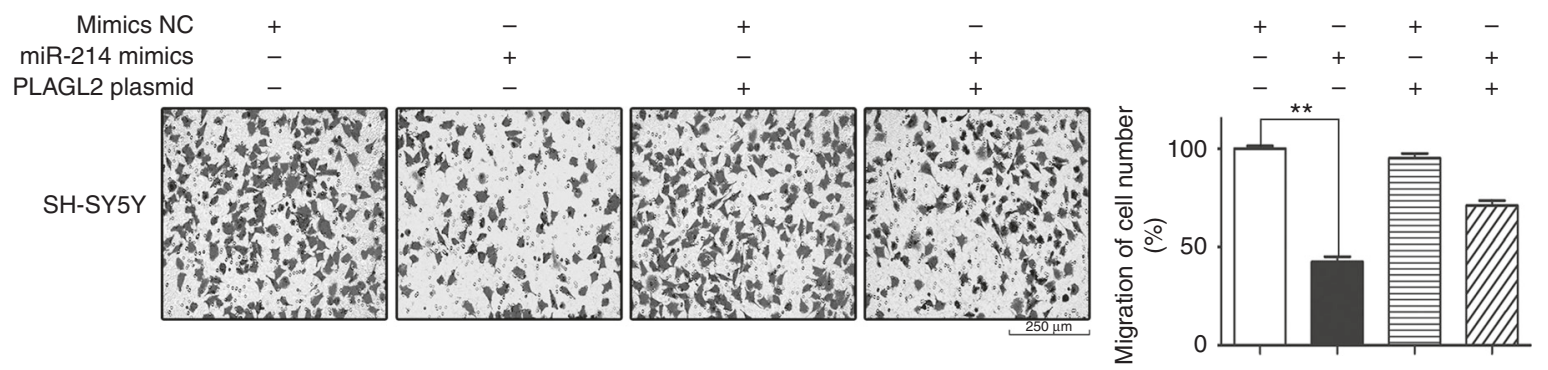

b
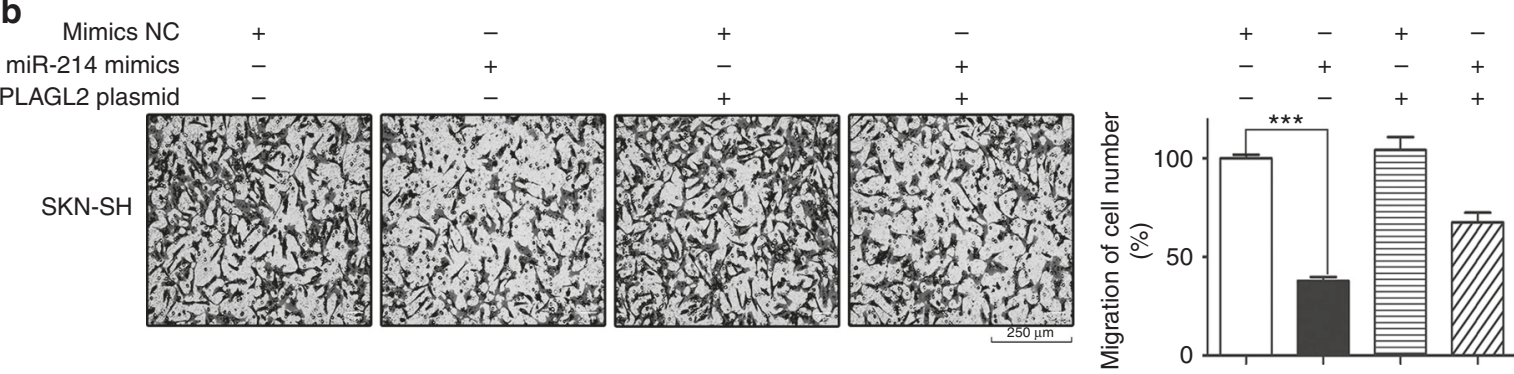

C

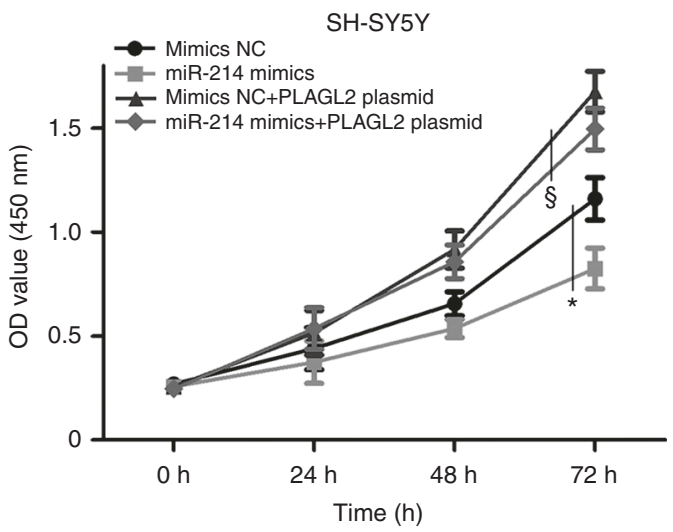

d

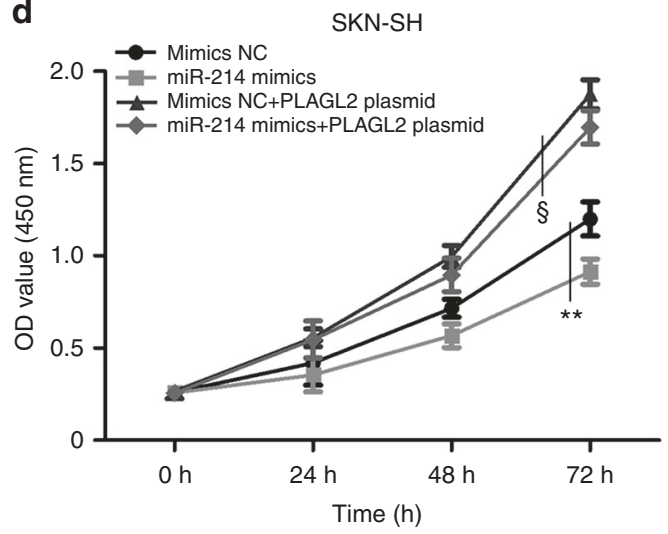

Fig. 6 Co-transfection of the PLAGL2 plasmid could partially remove the miR-214 mimic-mediated suppression in cell proliferation and migration. a-d The biological changes in migration and proliferation were partially reversed after co-transfection with miR-214 mimics and the PLAGL2 overexpression plasmid. We compared the co-transfected group with others and found that the migration and proliferation were partly reversed after co-transfection with miR-214 mimics and the PLAGL2 plasmid compared with the cells transfected with miR-214 mimics

PLAGL2 was found to be involved in cell proliferation and migration To further examine whether the PLAGL2 gene has a critical biological function in the development of HSCR, we employed PLAGL2 siRNA and an overexpression plasmid to knockdown and upregulate the expression of PLAGL2, respectively, in human SKN-SH and SH-SY5Y cells (Fig. 4e, f). Compared with the negative controls, the results from the treatment with PLAGL2 siRNA revealed a remarkably lower proliferation rate and a significant reduction in the number of migrated cells. Contrarily, transfection with the PLAGL2 overexpression plasmid notably increased the proliferation rate and number of migrated cells (Fig. 5a-d).

The co-transfection of the PLAGL2 plasmid could partially rescue miR-214-mediated cell proliferation and migration

In view of the fact that miR-214 could directly bind to three potential binding sites in the $3^{\prime}$ UTR region of PLAGL2 mRNA and causes remarkable inhibition against cell migration and proliferation, we carried out a series of rescue experiments to further verify whether miR-214 suppressed cell proliferation and migration by directly binding to the $3^{\prime}$ UTR region of PLAGL2 mRNA. Compared with cells transfected with miR-214 mimics alone, the co-transfection of the PLAGL2 plasmid and miR-214 mimics could partially draw up the miR-214 mimics-mediated suppression in cell proliferation and migration. The cell proliferation and migration abilities of the cells transfected with PLAGL2 plasmid were no less than the control (Fig. 6a-d). These data clearly indicated that the upregulation of miR-214 inhibited cell proliferation and migration by directly decreasing the expression of PLAGL2.

\section{DISCUSSION}

HSCR is a congenital gut motility disorder caused by the absence of the enteric neural crest cells (ENCCS). ${ }^{31}$ So far, two theories are used to explain the pathogenesis of HSCR. On one hand, during the 5th to 12th week of embryogenesis, ENCCs are unable to invade, proliferate, and migrate to the hindgut. ${ }^{32}$ On the other hand, some different studies revealed that the ENCCs could reach the distal gut but fail to proliferate or survive. ${ }^{31,33}$ Thus, any factors involved in the proliferation, migration, invasion, and survival of ENCCs could account for the development of HSCR. 
Increasing evidence indicates that the aberrant expression of miRNAs is closely associated with the initiation and/or progression of diverse diseases. For example, the miR-9-suppressed matrix metalloproteinase (MMP)-14 expression inhibits the angiogenesis, invasion, metastasis of neuroblastoma. ${ }^{34}$ The miRNA miR-558 induced the transcriptional activation of heparanase (HPSE) and then facilitates the tumorigenesis and aggressiveness of neuroblastoma (NB). ${ }^{35}$ Another molecule, miR-558, has also been shown to promote the progression of gastric cancer by targeting the HPSE promoter. $^{36}$ The increased expression of miR-214 in colon tissues upregulated nuclear factor-KB (NF-KB), which is associated with the development of ulcerative colitis (UC) and its progression to colorectal cancer (CAC). ${ }^{37}$ To date, no studies have revealed that miR-214 might be involved in the nosogenesis of HSCR.

In our study, miR-214 was found to be notably down-regulated in 24 HSCR patient samples and 20 matched controls, and PLAGL2 was confirmed to be a common target gene. PLAGL2 is a zinc-finger protein and a proto-oncogene involved in various tumors in humans. ${ }^{38,39}$ Enhanced PLAGL2 expression significantly suppressed glioma-initiating cell and neural stem cell (NSC) differentiation by activating the Wnt/B-Catenin pathway. ${ }^{22}$ To detect the possible role of miR-214 in the development of HSCR by binding to the target gene PLAGL2, human SKN-SH and SH-SY5Y cells were cultured and transfected with miR-214 mimics and negative controls. The expression levels of the PLAGL2 mRNA and protein were decreased, and the luciferase reporter assay revealed direct regulatory relationships between PLAGL2 and miR-214. Rescue experiments were performed using a PLAGL2 overexpression plasmid to partially counteract the inhibition of miR-214 on cell proliferation and migration.

These results suggest that the miR-214/PLAGL2 pathway is involved in the pathogenic mechanism of HSCR. However, the oneto-numerous and numerous-to-one interrelation between miRNAs and their target genes has been proven. Thus, further research is needed to verify whether any other genes participate in this signaling pathway. In our study, there were some limitations. For example, because of the limitation of the cell model of HSCR, the progression of ENCCs in this pathway is uncertain. Besides the sample size is relatively small. Therefore, additional studies are needed to demonstrate the pathogenesis of HSCR.

Taken together, our research demonstrates a novel mechanism in which enhanced miR-214 expression suppressed cell proliferation and migration by directly targeting PLAGL2 in two appropriate cell models. Our findings contribute to the novel understanding of HSCR pathogenesis and the creation of a new therapy target for HSCR in the future.

\section{ACKNOWLEDGEMENTS}

We are grateful to Dr. Shaotao Tang, the director of the Department of Pediatric Surgery of Union Hospital of Tongji Medical College for providing Hirschsprung Disease samples. We also thank Dr. Qiangsong Tong for giving us the two cell lines. This study was supported by the National Nature Science Foundation of China (No. 81470789 and No. 81271199). We also thank American Journal Experts (AJE) for proofreading this manuscript.

\section{AUTHOR CONTRIBUTIONS}

LW, WY and XS made substantial contributions to conception and design, acquisition of data, or analysis and interpretation of data; LW, WY, JC, ZZ, YS, JJ, ZL, and QT drafted the article and revised it critically for important intellectual content. LW, WY, $\mathrm{XZ}$, and $\mathrm{XS}$ gave the final approval of the version to be published.

\section{ADDITIONAL INFORMATION}

Competing interests: The authors declare no competing interests.

Publisher's note: Springer Nature remains neutral with regard to jurisdictional claims in published maps and institutional affiliations.

\section{REFERENCES}

1. Pan, Z. \& Li, J. Advances in molecular genetics of Hirschsprung's disease. Anat. Rec. 295, 1628-1638 (2012).

2. E. S. Emison, et al. A common sex-dependent mutation in a RET enhancer underlies Hirschsprung disease risk. Nature 434, 857-863 (2005).

3. Carter, T. C. et al. Hirschsprung's disease and variants in genes that regulate enteric neural crest cell proliferation, migration and differentiation. J. Hum. Genet. 57, 485-493 (2012).

4. McKeown, S. J. et al. Hirschsprung disease: a developmental disorder of the enteric nervous system. Wiley Interdiscip. Rev. Dev. Biol. 2, 113-129 (2013).

5. Tang, W. et al. Suppressive action of miRNAs to ARP2/3 complex reduces cell migration and proliferation via RAC isoforms in Hirschsprung disease. J. Cell. Mol. Med. 20, 1266-1275 (2016).

6. Lake, J. I. et al. Hirschsprung-like disease is exacerbated by reduced de novo GMP synthesis. J. Clin. Invest. 123, 4875-4887 (2013).

7. Heuckeroth, R. O. \& Schäfer, K. Gene-environment interactions and the enteric nervous system: Neural plasticity and Hirschsprung disease prevention. Dev. Biol. 417, 188-197 (2016).

8. Badner, J. A. et al. A genetic study of Hirschsprung disease. Am. J. Hum. Genet. 46, 568-580 (1990).

9. Zhang, Y., Kim, T. H. \& Niswander, L. Phactr4 regulates directional migration of enteric neural crest through PP1, integrin signaling, and cofilin activity. Gene Dev. 26, 69-81 (2012).

10. Chatterjee, S. et al. Enhancer variants synergistically drive dysfunction of a gene regulatory network in Hirschsprung disease. Cell 167, 355-368 (2016).

11. Jiang, Q. et al. Functional loss of semaphorin $3 C$ and/or semaphorin $3 D$ and their epistatic interaction with ret are critical to Hirschsprung disease liability. Am. J. Hum. Genet. 96, 581-596 (2015).

12. Fadista, J. et al. Genome-wide association study of Hirschsprung disease detects a novel low-frequency variant at the RET locus. Eur. J. Hum. Genet. 26, 561-569 (2018).

13. Huntzinger, E. \& Izaurralde, E. Gene silencing by microRNAs: contributions of translational repression and mRNA decay. Nat. Rev. Genet. 12, 99-110 (2011).

14. Bartel, D. P. MicroRNAs: genomics, biogenesis, mechanism, and function. Cell 116, 281-297 (2004).

15. Sharan, A. et al. Down-regulation of miR-206 is associated with Hirschsprung disease and suppresses cell migration and proliferation in cell models. Sci. Rep. 5, 9302 (2015).

16. Q. Wu, et al., MiR-221-3p targets ARF4 and inhibits the proliferation and migration of epithelial ovarian cancer cells. Biochem. Biophys. Res. Commun. 497, 1162-1170 (2018).

17. Wang, P. et al. MicroRNA-126 increases chemosensitivity in drug-resistant gastric cancer cells by targeting EZH2. Biochem. Biophys. Res. Commun. 479, 91-96 (2016).

18. Qiang, R. et al. Plexin-B1 is a target of miR-214 in cervical cancer and promotes the growth and invasion of HeLa cells. Int. J. Biochem. Cell. Biol. 43, 632-641 (2011).

19. Penna, E., Orso, F. \& Taverna, D. miR-214 as a key hub that controls cancer networks: small player, multiple functions. J. Invest. Dermatol. 135, 960-969 (2015).

20. Hanks, T. S. \& Gauss, K. A. Pleomorphic adenoma gene-like 2 regulates expression of the p53 family member, p73, and induces cell cycle block and apoptosis in human promonocytic U937 cells. Apoptosis 17, 236-247 (2012).

21. Sekiya, R. et al. PLAGL2 regulates actin cytoskeletal architecture and cell migration. Carcinogenesis 35, 1993-2001 (2014).

22. Zheng, $H_{\text {., }}$ et al. PLAGL2 regulates Wnt signaling to impede differentiation in neural stem cells and gliomas. Cancer Cell. 17, 497-509 (2010).

23. Zhu, J. J. et al. HOXB5 binds to multi-species conserved sequence (MCS+9.7) of RET gene and regulates RET expression. Int. J. Biochem. Cell. Biol. 51, 142-149 (2014).

24. Cai, P., et al. Aberrant expression of LncRNA-MIR31HG regulates cell migration and proliferation by affecting miR-31 and miR-31* in Hirschsprung's disease. J. Cell. Biochem. 119, 8195-8203 (2018).

25. Chen, $G$. et al. LncRNA AFAP1-AS functions as a competing endogenous RNA to regulate RAP1B expression by sponging miR-181a in the HSCR. Int. J. Med. Sci. 14, 1022-1030 (2017).

26. Xiang, $X$. et al. miRNA-584-5p exerts tumor suppressive functions in human neuroblastoma through repressing transcription of matrix metalloproteinase 14 . Biochim. Biophys. Acta 1852, 1743-1854 (2015).

27. Jeong, S., Oh, J. M., Oh, K. \& Kim, I. Differentially expressed miR-3680-5p is associated with parathyroid hormone regulation in peritoneal dialysis patients. PLoS One 12, e170535 (2017).

28. Sharan, A., et al. Down-regulation of miR-206 is associated with Hirschsprung disease and suppresses cell migration and proliferation in cell models. Sci. Rep. 5, 9302 (2015). 
29. Zhou, B. et al. Downregulation of miR-181a upregulates sirtuin-1 (SIRT1) and improves hepatic insulin sensitivity. Diabetologia 55, 2032-2043 (2012).

30. Jiang, M. et al. Calretinin, S100 and protein gene product 9.5 immunostaining of rectal suction biopsies in the diagnosis of Hirschsprung' disease. Am. J. Transl. Res. 8, 3159-3168 (2016)

31. Langer, J. C. Hirschsprung disease. Curr. Opin. Pediatr. 25, 368-374 (2013).

32. Lake, J. I. \& Heuckeroth, R. O. Enteric nervous system development: migration, differentiation, and disease. Am. J. Physiol. Gastrointest. Liver Physiol. 305, G1-G24 (2013).

33. Shu, X. et al. Treatment of aganglionic megacolon mice via neural stem cell transplantation. Mol. Neurobiol. 48, 429-437 (2013).

34. Zhang, $\mathrm{H}$. et al. microRNA-9 targets matrix metalloproteinase 14 to inhibit invasion, metastasis, and angiogenesis of neuroblastoma cells. Mol. Cancer Ther. 11, 1454-1466 (2012).

35. $\mathrm{Qu}, \mathrm{H}$. et al. miRNA-558 promotes tumorigenesis and aggressiveness of neuroblastoma cells through activating the transcription of heparanase. Hum. Mol. Genet. 24, 2539-2551 (2015).

36. Zheng, L. et al. miRNA-558 promotes gastric cancer progression through attenuating Smad4-mediated repression of heparanase expression. Cell Death Dis. 7, e2382 (2016)

37. Polytarchou, C. et al. MicroRNA214 is associated with progression of ulcerative colitis, and inhibition reduces development of colitis and colitis-associated cancer in mice. Gastroenterology 149, 981-92.e11 (2015).
38. Castilla, L. H., et al. Identification of genes that synergize with Cbfb-MYH11 in the pathogenesis of acute myeloid leukemia. Proc. Natl Acad. Sci. USA 101, 4924-4929 (2004).

39. Furukawa, T. et al. Involvement of PLAGL2 in activation of iron deficient- and hypoxia-induced gene expression in mouse cell lines. Oncogene 20,4718-4727 (2001).

Open Access This article is licensed under a Creative Commons Attribution-NonCommercial-NoDerivatives 4.0 International License, which permits any non-commercial use, sharing, distribution and reproduction in any medium or format, as long as you give appropriate credit to the original author(s) and the source, and provide a link to the Creative Commons license. You do not have permission under this license to share adapted material derived from this article or parts of it. The images or other third party material in this article are included in the article's Creative Commons license, unless indicated otherwise in a credit line to the material. If material is not included in the article's Creative Commons license and your intended use is not permitted by statutory regulation or exceeds the permitted use, you will need to obtain permission directly from the copyright holder. To view a copy of this license, visit http://creativecommons.org/licenses/by-nc-nd/4.0/.

(c) The Author(s) 2019 